TTR

Traduction, terminologie, re?daction

\title{
Pour une historiographie des théories de la traduction : questions de méthode
}

\section{Lieven D’hulst}

Volume 8, numéro 1, 1er semestre 1995

Orientations européennes en traductologie

URI : https://id.erudit.org/iderudit/037195ar

DOI : https://doi.org/10.7202/037195ar

Aller au sommaire du numéro

\section{Éditeur(s)}

Association canadienne de traductologie

ISSN

0835-8443 (imprimé)

1708-2188 (numérique)

Découvrir la revue

\section{Citer cet article}

D’hulst, L. (1995). Pour une historiographie des théories de la traduction : questions de méthode. TTR, 8(1), 13-33. https://doi.org/10.7202/037195ar

\section{Résumé de l'article}

Pour une historiographie des théories de la traduction : questions de méthode - L'historiographie de la traduction et des théories de la traduction est un secteur sous-développé de la traductologie, tant dans la perspective de l'interdisciplinarité que celle-ci réclame et dont on trouve des exemples en particulier dans l'histoire de la discipline que dans l'intérêt grandissant pour une compréhension plus approfondie, en termes historiques, des relations étroites entre les théories modernes spécialement en prenant en compte leurs origines et leur développement. L'article aborde plusieurs problèmes méthodologiques de la recherche historique en théorie de la traduction. En premier lieu, on insiste sur la nécessité de fonder l'analyse sur des corpus exhaustifs, ce qui implique de prendre en considération des sources traditionnellement négligées ainsi que les disciplines connexes. En second lieu, on plaide ici en faveur d'une interaction réfléchie entre concepts contemporains et historiques en faisant jouer à ces derniers un rôle complémentaire et non pas de substitut. Les relations entre contenu et contexte peuvent être décrites selon le même principe. En troisième lieu, l'article analyse certains problèmes que posent la nature évolutive des théories et leur constitution en " paradigmes ". Enfin, le modèle de progrès prédominant dans les théories contemporaines de la traduction est critiqué et confronté à une perspective sur l'évaluation déterminée par des facteurs historiques proprement dits parmi lesquels le facteur contextuel qui fait ici l'objet d'une analyse particulière.

Tous droits réservés @ TTR: traduction, terminologie, rédaction — Les auteurs, Ce document est protégé par la loi sur le droit d’auteur. L’utilisation des 1995

services d'Érudit (y compris la reproduction) est assujettie à sa politique d'utilisation que vous pouvez consulter en ligne.

https://apropos.erudit.org/fr/usagers/politique-dutilisation/ 


\section{Pour une historiographie des théories de la traduction: questions de méthode}

\section{Lieven D'hulst}

\section{Introduction}

À mesure que la traductologie tend à s'ériger en une discipline autonome ou, mieux, une interdiscipline, la question de son identité propre reste à l'ordre du jour, si elle ne met pas en péril un équilibre à défendre et donc ressenti comme précaire (voir par exemple $\mathrm{M}$. Snell-Hornby, 1988). Car, en théorie et en pratique, l'interdisciplinarité suppose un alliage subtil entre ce qui est singulier et ce que l'on peut partager, et il n'est pas sûr qu'aussi bien les traductologues que leurs partenaires issus d'autres disciplines puissent s'accorder sur la nature et le contenu d'un tel dosage.

Mais quoi qu'il en soit de ce dernier, la pertinence de la question qu'on vient de poser ne peut être mise en doute, face à la conviction largement répandue que l'alliance avec plusieurs de ses anciennes concurrentes (linguistiques et comparatistes, par exemple), conditionnera les progrès futurs de la discipline. En contrepoint, la prospective traductologique semble ignorer la pratique séculaire d'une réflexion sur la traduction qui n'a eu de cesse de chercher à trouver un fondement rationnel à un objet imprécis, partagé par diverses disciplines, alors même qu'elle a parallèlement emprunté à celles-ci concepts et méthodes. Hier comme aujourd'hui, la répartition en théories, méthodes, analyses, histoires, applications, etc. reproduit celle qu'ont adoptée les sciences reposant sur une 
épistémologie alors commune. Les interférences entre les disciplines, à propos de la modélisation de telle ou telle de ces tâches, n'ont pas toujours été acceptées sans peine par les générations successives de traductologues; pourtant, elles sont hautement caractéristiques à la fois de l'histoire et de la situation présente des théories. Plaider comme on le fait de nos jours pour la nécessité d'une traductologie enfin interdisciplinaire, c'est non seulement occulter la tradition, mais c'est s'inscrire à son tour dans l'histoire de la discipline en exprimant l'un de ses grands thèmes récurrents (voir par exemple $\mathrm{R}$. Copeland, 1991).

Le petit exercice critique auquel nous venons de nous livrer est sans doute en partie contestable; il n'a d'autre but que de rappeler la relation difficile entre théorie et histoire et de permettre à ce sujet la formulation de l'hypothèse suivante: plus la mémoire théorique s'estompe dans les efforts de conceptualisation présentés comme originaux et propres à notre époque ${ }^{1}$, plus la perspective historique aura intérêt à souligner les sources antérieures de ce savoir.

Mais, au delà de cette dimension critique, quel propos tenir sur la place et les fonctions de l'histoire des théories, laquelle, par la vertu d'un certain flou, se prête bien au rôle du cheval de Troie au sein de la traductologie? Il est prévisible, par exemple, que l'historien arguera de cas comme celui que nous venons de citer pour mieux étayer sa propre position vis-à-vis des autres disciplines. Or, qu'il brigue celle d'arbitre, ou de juge des «progrès réels») accomplis par ces disciplines, il ne doit pas pour autant s'attribuer le poste d'observateur neutre des mécanismes d'un jeu auquel il ne prendrait pas part: à son tour, et non moins que les théoriciens, par exemple, il est impliqué dans un débat, tant sur les concepts et les méthodes, que sur les fonctions de la recherche historique. Et ce débat n'échappe pas davantage à la tentation anhistorique qu'il croit percevoir chez les théoriciens de la traduction.

1. Au point de situer la naissance de la discipline, selon la doxa, aux lendemains de la Deuxième Guerre mondiale. 
On est bien obligé d'admettre que les questions historiques sont d'une grande complexité. Et elles n'ont guère encore retenu l'attention systématique des traductologues, ni même de nombreux historiens de la traduction. L'on s'imagine mal dès lors pourquoi et comment l'esprit interdisciplinaire, si puissant ailleurs, $n$ 'inspirerait pas, en l'occurrence, le recours à la linguistique, aux études littéraires ou à la philosophie, qui se sont en quelques décennies dotées d'une tradition solide en matière d'histoire des théories. Bien entendu, il faudra, le cas échéant, définir avec soin et prudence les procédures de transfert (voir Lambert, 1993); de même il faudra investir sérieusement sur le plan de l'épistémologie et des méthodes historiques appliquées aux théories de la traduction. Nous sommes loin du compte. Dans ce qui suit, nous nous bornerons à une modeste suite de réflexions sur quelques points de méthode ${ }^{2}$.

\section{Du bon usage des sources}

La nature de la recherche historique telle que nous la souhaitons, fondée en théorie et en méthode, est proprement désignée par le terme «historiographie», terme qui a fait son chemin en histoire de la linguistique (Koerner, 1989, pp. 51-56). Ce qui ne signifie pas que la communauté scientifique adoptera sans peine une définition universelle et une démarche unique. Bien au contraire: un accord peut sembler aisé entre historiens de la traductologie sur le choix entre une écriture de type positiviste, simple inventaire et chronique des faits, et celle qui plus spécifiquement rend compte des concepts théoriques et de leur agencement discursif; par contre, il n'en est pas de même lorsqu'il s'agit de privilégier, dans l'étude de la communication traductologique, les textes théoriques, ou leurs auteurs, ou leur réception, ou leur contextualisation. Au même titre, s'avère délicate l'option pour un modèle discursif narratif, herméneutique, analytique, etc.

2. Elles forment le complément d'autres contributions auxquelles on se permet de renvoyer le lecteur (D'hulst, 1990, 1991, 1993, 1995). 
Cela étant, il est vain d'insister sur les avantages respectifs des différentes options, aussi longtemps que celles-ci ne sont pas mises à l'épreuve dans la pratique historiographique. Corrélativement, une certaine pluralité dans les applications apparaîtra comme l'expression spontanée de l'intérêt pour les questions d'histoire, et préviendra peut-être les rares énergies disponibles de s'enliser dans un débat qui risque rapidement de devenir stérile faute de références suffisantes aux données factuelles.

L'étude historique des théories de la traduction présuppose notamment l'inventaire exhaustif des textes qui en sont l'expression, autrement dit l'achèvement de l'étape heuristique. Or, notre savoir est si lacunaire que toute affirmation à portée générale relève en fait du simple a priori; c'est le cas du propos suivant:

Ce discours [...] est d'une surprenante minceur: peu d'ouvrages, une masse de notes, de lettres, de préfaces, etc. et si l'on compare ce corpus à celui des textes «critiques» que la littérature a produit, disons, depuis le $\mathrm{XVI}^{e}$ siècle, on devra en conclure que les traducteurs sont fort parcimonieux lorsqu'ils parlent de leur activité. (Berman, 1993, p. 40)

La thèse de Berman devrait subir une contre-épreuve: la «minceur» ne doit pas être mesurée seulement par rapport au discours contemporain, mais par rapport aux discours sur la langue, la littérature, etc. Est-on bien certain d'aboutir à des conclusions différentes?

En vérité, force est de constater que nous manquons de bibliographies exhaustives, sauf pour de rares cultures et certaines périodes, que les textes sont peu connus et peu accessibles. D'où le recours presque exclusif, lorsqu'elles existent, à des anthologies de fragments souvent très courts, qu'on estime représentatifs de la variété, en synchronie et en diachronie, des théories étudiées. Ces

3. Le fait que l'auteur ne tienne pas compte de cette possibilité laisse transparaître son attitude critique, plutôt qu'historique, envers le passé de la discipline. 
fragments sont extraits de leurs contextes, cependantqu'ils possèdent des propriétés génériques différentes suivant qu'il s'agit de traités, de préfaces, de comptes rendus, de lettres, etc. Leur transparence risque aussi d'être trompeuse, si l'on ne prend pas en considération les contraintes discursives qui pèsent sur l'expression, et gèrent la structure des théories, le choix des images et des exemples.

D'autres sources restent à ce jour négligées: les correspondances de traducteurs et de critiques, les méthodes d'enseignement des langues, les manuels et traités de traduction, les versions manuscrites ou les rééditions avec variantes de textes déjà publiés. Elles peuvent éclairer la conceptualisation historique, la lisibilité, la diffusion des textes, les contraintes institutionnelles, voire la censure, ainsi que la part individuelle ou «biographique» d'une pensée théorique. Les textes publiés sont d'abord la fixation graphique d'une pensée en mouvement. L'exemple suivant montre comment la juxtaposition de textes différents d'un même auteur permet de saisir ce dynamisme, en l'occurrence l'influence sur les idées traductologiques du genre utilisé.

Charles Loyson, poète français, publiciste, professeur et traducteur, soutient en 1813, devant l'Académie de Paris, une thèse de doctorat sur la traduction de la poésie antique. Nous disposons d'une lettre adressée peu avant la soutenance à l'un de ses mentors, où le sujet de la thèse est évoqué en des termes surprenants, et fort éloignés des idées finalement retenues dans la version publiée de la thèse:

Homère est [...] intraduisible en vers. Eh bien! j'ajouterai, si vous voulez, la prose française. Et je crois que pour le rendre il faut une traduction presque interlinéaire, une traduction qui serait un monstre, considérée comme un ouvrage original, semblable, enfin, à ces traductions latines si barbares, qu'on lit avec plus de plaisir que la prose travaillée et brillantée de $\mathrm{M}$. Lebrun, parce qu'elles nous donnent au moins une idée d'Homère et des moeurs de son temps. Tout cela sent un peu le paradoxe. (Séché, 1905, p. 293)

Ce point de vue controversé était particulièrement incompatible avec les idées néo-classiques sur la traduction qui avaient cours au sein 
de la nouvelle université impériale, créée en 1808. Loyson a senti le paradoxe: les maîtres dont dépendait sa carrière future souhaitaient encourager et généraliser la pratique de la traduction «élégante», à mi-chemin entre les ambitieuses traductions littéraires selon la tradition de Delille, et les versions scolaires dépourvues des atours indispensables à l'écriture rhétorique classique réimposée dans l'enseignement. Par delà, il s'agissait de restaurer la place et le prestige des langues anciennes, gravement atteints par les réformes de l'enseignement proposées par la Convention, lesquelles accordaient la prééminence au français, langue nationale, et avaient pratiquement supprimé la rhétorique dans le cursus scolaire. D'où la recherche de compromis prudents dont nous trouverons un écho dans la version publiée de la thèse.

Par ailleurs, en constituant le corpus de textes qu'il se propose d'étudier, l'historien doit glaner au delà du domaine proprement traductologique, en particulier du côté des disciplines voisines. Ainsi, les poétiques, rhétoriques, grammaires et méthodes des langues des $\mathrm{XVII}^{e}$ et XVIII ${ }^{e}$ siècles français reflètent un intérêt inégal pour les règles et principes de l'art de la traduction: souvent absentes des trois premières, les réflexions se cantonnent en partie dans les méthodes de latin; en partie aussi, elles échappent aux formes traditionnelles de la théorie, en adoptant celle de la préface ou du compte rendu (D'hulst, 1990, pp. 103-106 et 173-176). Mais à la fin du XVIII ${ }^{e}$ siècle, elles réapparaissent dans les grammaires générales des Idéologues inspirées de Condillac, sous une forme métaphorique désignant des modes de transposition entre les sciences définies comme des «langages», ou entre les systèmes de signes au sein d'un même langage: des sons aux lettres par exemple; ou encore, de l'écriture à la lecture ${ }^{4}$. Comment sont agencées les conceptions linguistique et intersémiotique de la traduction, avant que la pensée traductologique ne devînt au $\mathrm{XIX}^{\mathrm{e}}$ siècle la chasse gardée des traducteurs-professeurs et quelques traducteurs-poètes: voilà une question qui nécessite un constant aller et retour entre l'objet et sa contextualisation.

4. Voir D’hulst (1993, pp. 93-94). 
Sous quelque forme qu'ils se présentent, les textes requièrent un traitement circonspect, non seulement parce que le chercheur se doit de privilégier leur conceptualisation historique, mais également parce que ce sont des expressions spécifiques d'un savoir aux présupposés sous-jacents. La reconstruction de ces derniers est forcément hypothétique, d'autant plus qu'il peut s'agir de savoirs inconsciemment informulés, de savoirs censurés, même de savoirs oubliés. Le non-dit évoqué par Michel Foucault (Foucault, 1966) doit donc être défini sur une toile de fond élargie, l'épistémè de l'époque qui permettra dans le meilleur des cas d'avancer de prudentes hypothèses quant au contenu de ces présupposés. Comme exemples, on peut se référer ici aux articles encyclopédiques sur la traduction: leur examen comparé sur une période suffisamment longue, qu'on peut faire commencer par l'Encyclopédie, révélera des écarts que n'expliquent pas assez les différences des théories. Ainsi, comment s'est opérée, dans un espace forcément restreint, la sélection des informations? Est-elle le fait exclusif du rédacteur? Et comment celui-ci a-t-il été désigné? Quelles ont été envers lui les consignes de ses commanditaires, le ou les éditeurs scientifique et commercial?

\section{Contenus et contextes}

L'objectif, premier et essentiel à nos yeux, que devrait se fixer l'historien des théories de la traduction est leur reconstruction optimale selon le point de vue de leurs concepteurs et de leurs usagers. Or, dans quelle mesure le recours à des concepts modernes peut-il servir la reconstruction des concepts historiques de la traduction? Il est difficile d'éviter une telle question lorsqu'on veut s'assurer l'appui de théories de l'historiographie, compte tenu en particulier du haut degré de complexité des phénomènes historiques qu'il s'agit d'observer. Mais faut-il accorder un rôle similaire aux modèles traductologiques? L'histoire de la linguistique, quant à elle, a invoqué cette complexité pour souligner la nécessité d'un croisement de plusieurs démarches:

[...] various descriptive models are applicable to the history of linguistics, precisely because the history of linguistics has a 
multidimensional aspect. Linguistic history is not a matter of an evolution of ideas along one time axis, but is a complex bundle of developments (of ideas and of techniques) along various time axes. (Swiggers, 1990, p. 27)

En envisageant les implications méthodologiques, il importe évidemment de se prémunir contre les dangers inhérents au rapprochement du passé et du présent, le plus apparent étant une attitude "doxographique» vis-à-vis des concepts historiques, qui consiste à les choisir, à les décrire et surtout à les évaluer d'après l'étalon de la science contemporaine. À l'opposé, l'historien qui réduirait son rôle au seul fait d'enregistrer viendrait à manquer les effets du dynamisme herméneutique, d'une «sympathetische Annäherung» (Schlieben-Lange, 1991, p. 313), participant à l'effort de compréhension de théories éloignées dans le temps. A plus forte raison, il ne pourrait, à l'endroit de celles-ci, appliquer le principe suivant:

We look to find and to preserve a certain level of consistency between our attitudes and acts. If inconsistency comes to our notice then we attempt to resolve such inconsistency. The principle of charity simply extends this into a general principle of interpretation - we ought to treat others as we treat ourselves. We should seek the same overall consistency in our interpretation of other speakers, actors or believers as we do with respect to ourselves. (Malpas, 1988, p. 25)

Corrélativement, la fonction des concepts modernes est complémentaire des concepts historiques et ne peut leur être substituée. Plutôt que de couler ces derniers dans des moules qui risquent de déformer la perception d'époque, il y aurait intérêt à les affecter à la description - soit de la part restée implicite dans la conceptualisation historique, soit de propriétés théoriques plus générales, soit encore de processus qui assurent l'interrelation entre les théories de la traduction et leur contextualisation.

Voyons un exemple relatif aux caractéristiques générales de la formation des théories. La description telle quelle de ces dernières suscite en effet une interrogation sur leur naissance, sur leur 
développement et transformation, souvent au travers de contacts avec d'autres disciplines ou sous l'effet de contraintes externes. La psychologie cognitive a développé en l'occurrence un modèle d'analyse fondé sur l'observation détaillée (et renouvelée dans des conditions historiques variables, portant sur plusieurs disciplines), d'un processus d'extension métaphorique de concepts de base propres à une discipline: cette extension s'obtient par l'association de ces concepts à d'autres, désignant des pratiques différentes, donnant lieu à ce que Max Black appelle des «archétypes»:

By an archetype I mean a systematic repertoire of ideas by means of which a given thinker describes, by analogical extension, some domain to which those ideas do not immediately and literally apply. (Black, 1972, p. 241)

Un archétype s'exprime en une suite de mots-clefs et d'expressionsclefs prenant la forme de définitions ou propositions énonciatives, telles que «la traduction est un processus de communication». Lorsque s'opère un transfert plus systématique, un modèle ainsi qu'une théorie peuvent en résulter.

Les vues de Black ont été appliquées par Raymond van den Broeck à l'analyse du processus de la traduction en termes de jeu, analyse conçue par Jiři Levý et adoptée ensuite par plusieurs chercheurs, dont James Holmes: «translation is a decision process)" (1967). Il va de soi que Levý, comme d'autres avant lui, n'ignoraient pas que toute traduction implique des «choix», selon une terminologie usuelle. Mais c'est le repérage dans la théorie du jeu d'une série de concepts de base appropriés et formant réseau qui a déterminé leur transfert au domaine traductologique: ainsi des concepts comme "consecutive situations», «decision», "selective instructions», «minimax strategy» ont résulté en un nouveau modèle du processus de la traduction. Et ce modèle a été développé en une théorie générale grâce à des emprunts à la sémiotique, permettant la distinction au sein du processus traductif d'une triple dimension: sémantique, syntaxique et pragmatique. 
Que nous enseigne un tel exercice? En d'autres termes, qu'apporte à l'analyse de la formation d'une théorie une discipline comme la psychologie cognitive? Sommes-nous désormais en mesure d' «expliquer» de manière satisfaisante la naissance d'une théorie comme celle de Levý? La réponse est prudemment affirmative à une condition près ${ }^{5}$ : que l'historien choisisse clairement sa démarche en fonction de son projet de lecture. Or, si ce dernier, comme c'est le cas en l'occurrence, se propose la reconstruction optimale du champ conceptuel d'un théoricien d'époque, ses ambitions doivent se limiter à cet objet précis, laissant ouverte la voie à d'autres démarches qui procéderont à une sélection et analyse différentes d'objets découpés dans le même champ réflexif.

À plus long terme, on peut envisager l'articulation de plusieurs démarches, celles, par exemple, qui portent sur les contenus théoriques et sur leurs contextes. Elle est toutefois délicate. Prenons l'exemple du concept «copie servile» largement diffusé à l'Âge Classique. On imagine sans peine le lien qui rattache ce concept à la théorie imitative des Beaux-Arts. D'où la tendance spontanée à concevoir alors une base théorique commune. Mais estil souhaitable de définir celle-ci de manière aussi globale, sans étudier en premier lieu, et de manière exhaustive, le réseau conceptuel traductologique auquel se rattache peut-être plus intimement l'expression "copie servile»? Par ailleurs, un autre péril existe qui consiste à traiter comme une «transformation» tout écart conceptuel entre l'art de la traduction et les principes des BeauxArts.

De manière plus générale, les corrélations complexes entre les théories de la traduction et des champs comme l'idéologie, la littérature, la religion, les institutions, etc. invitent à formuler des hypothèses en tous points provisoires en l'absence d'études de cas

5. Il est superflu de s'appesantir sur le principe de relativisme historique, qui s'applique évidemment ici comme ailleurs. 
suffisamment nombreuses et systématiques ${ }^{6}$. On ne doit pas dès lors s'étonner que le traductologue contemporain ait souvent peine à dissimuler son irritation devant ce qui lui paraît être une sempiternelle répétition de problèmes mieux définis et résolus aujourd'hui:

Whenever one takes the trouble to peruse the hundreds of publications on translation theory and practice today and in former times, one cannot help being assailed by a feeling of frustration. The same problems and the same affirmations about the same problems are repeated again and again: whether it is better to translate literally or freely according to the meaning (or sense) of words and sentences or texts, whether form or meaning (content) of a source text are more important, whether rhyme and rhythm are to be preserved or substituted and so on. [...] Has there been no progress in all these centuries of hard work? (Vermeer, 1994, p. 3)

Non seulement le traductologue prend ainsi la place de l'historien, pratiquant un facile anachronisme, mais il réduit son objet aux textes proprement dits, négligeant de la sorte l'interaction avec le contexte qui aide à restituer à ces textes leur signification. La séduction tenace en effet d'enlever, au travers de parallèles faciles avec notre univers intellectuel, certaine spécificité de la pensée historique peut et doit être combattue par une attention soutenue aux facteurs contextuels.

\section{Sur l'évolution}

On peut penser que le fait historique est interprété selon une évolution observable dans le temps, autant sinon davantage que par le fait qu'il appartient au passé. Pour l'historien, le facteur temporel est des plus complexes: il comprend la constitution, le développement, la transformation, la simplification, la transmission,

6. Une exception: l'étude déjà citée de R. Copeland (1991). Sur la relation entre contexte et cognition, voir E. Elffers-van Ketel (1991, pp. 158-162). 
la mémoire et l'oubli de faits historiques, en l'occurrence des théories de la traduction. Ces questions n'affleurent qu'indirectement le travail de l'historien lorsqu'il s'agit de structurer un corpus selon une certaine périodisation. Il adoptera les grandes divisions de l'histoire culturelle (Antiquité, Moyen Âge, Renaissance, Classicisme, etc.), ou bien il distinguera de grands axes théoriques qui lui paraissent globalement dominer des périodes plus souplement distinguées: la théorie de l'imitation, celle de la traductionappropriation, de la traduction créatrice, etc. (voir Ballard, 1992).

Pour rendre compte des processus cités, il faut surtout d'une part s'attacher à l'agencement dans le temps des théories qui se côtoient et se concurrencent, et d'autre part veiller à la dimension temporelle inégale où s'inscrivent dans chaque théorie les différents concepts. Sur ce point, la distinction introduite par Fernand Braudel entre la longue durée (niveau structurel), la moyenne durée (le niveau conjoncturel) et la courte durée (le niveau événementiel), pourrait se révéler utile ${ }^{7}$. Appliquée au $X V I I^{e}$ siècle français, elle nous permettrait de mieux comprendre l'interaction et la concurrence entre diverses conceptions traductologiques, précisant les vues de Rener (1989) sur la constitution dans la longue durée de la tradition occidentale du concept classique d'interpretatio. Ainsi, s'inscriraient dans la moyenne durée des questions cycliques, comme la recherche des universaux de la traduction, la constitution d'une pensée traductologique autonome, les rapports hiérarchiques entre languesource et langue-cible. La courte durée, enfin, permettrait de confronter les différentes conceptions de la traduction: celle de $\mathrm{Du}$ Marsais, Pluche, Batteux, Beauzée, Marmontel, Saint-Simon, etc.

Un double avantage est attaché à la distinction en de tels niveaux: on recourt au même paramètre interne, plutôt que d'en emprunter un ou plusieurs à l'histoire culturelle, littéraire, linguistique ou autre; chaque niveau est articulé à l'autre, et ne peut être compris isolément. Des formes affinées qui relient

7. Cette distinction a été proposée en histoire de la linguistique (Swiggers, 1990, p. 28). 
l'événementiel et le conjoncturel sont notamment proposées par les études de Copeland (1991) et de L. Korpel (1992)

Ces avantages ressortent mieux encore lorsqu'on connaît l'écueil suivant, généralement sous-estimé en histoire de la traductologie: il peut arriver qu'un ensemble d'assertions théoriques soient réunies par l'historien en un savoir plus ou moins homogène, et transmis de siècle en siècle $e^{8}$, sous une forme simplifiée d'où finissent par disparaitre les nuances et changements. Un nombre appréciable d'introductions modernes à la science de la traduction passent sous silence la complexe structure argumentative ainsi que la contextualisation des savoirs traductologiques du passé, en suggérant indûment la possible lecture transparente de ces textes ${ }^{9}$.

Le lien entre les propriétés internes des théories de la traduction et la composition en groupes de recherche, voire en "écoles», des chercheurs qui se réclament des mêmes théories requiert un examen minutieux. Lorsqu'entre ces chercheurs s'établit une certaine cohésion, le cas échéant encouragée par les institutions où ils sont affiliés, cohésion qui s'exprime notamment en des projets collectifs, il y a lieu de se référer au concept de "paradigme» dans le sens défini par Kuhn (1970). Les «descriptive translation studies» constituent un exemple intéressant d'une telle évolution: l'expression citée a été diffusée par James Holmes à partir de 1972 (Holmes, 1988 [1972]) avant d'être adoptée, et intégrée à plusieurs démarches scientifiques mises en oeuvre par un nombre limité de chercheurs, surtout néerlandais, belges et israéliens, au cours des années 1970 et 1980. La reconnaissance internationale de ce que ces chercheurs présentaient eux-mêmes comme une contribution originale à la traductologie a rapidement donné lieu à l'idée d'une vision unifiée de chercheurs désormais constitués en école (la «manipulation schooly selon M. Snell-Hornby, 1988, p. 22 et sv.): des

8. Qualifié de «Wissensbestand», selon B. Schlieben-Lange (1989, p. 14).

9. Un exemple récent: Stolze (1994). 
convergences de théorie et de méthode ont été interprétées comme un savoir homogène, disposant aussi d'une expression canonique ${ }^{10}$.

Des représentants du paradigme apparent ont pris position à l'égard de ce savoir, à leurs yeux incompatible avec la dynamique propre à leurs travaux, au point de susciter une forme d'historiographie soit critique soit apologétique. Des exemples nous sont donnés par plusieurs contributions au colloque sur James Holmes tenu à Amsterdam en 1991 (van Leuven-Zwart et Naaijkens, 1991): les efforts pour renforcer les caractéristiques communes reposant sur un sens profond de la tradition y alternent avec la mise en relief de différences, voire la reconnaissance de ruptures avec cette tradition.

\section{La question de la valeur}

L'un des grands axiomes de la traductologie contemporaine veut que la discipline ait accompli un progrès rapide depuis la seconde moitié de notre siècle, progrès qui tranche brutalement avec un passé «préscientifique». Ce jugement de valeur n'est pas davantage fondé que l'axiome dont il fait partie. Aux yeux de l'historien, en contrepartie, ce passé se dérobe à la généralité du propos comme elle se dérobe à sa fixation critique. L'évolution de la pensée traductologique ne suit pas, en effet, un chemin rectiligne et prévisible, de même que les appréciations des théories antérieures sont à leur tour sujettes à des changements. Voire: si les jugements de valeur sont régulièrement adaptés à de nouvelles conceptions, le progrès peut consister en un «retour en arrière». Ainsi des théoriciens contemporains peuvent redécouvrir des ancêtres, tels Benjamin ou Derrida réhabilitant la pensée de Schleiermacher. Enfin, le savoir historique lui-même peut en sens inverse servir

10. Il est significatif que la source majeure, sinon unique, de plusieurs commentateurs des «Descriptive Translation Studies», soit l'introduction par Theo Hermans à un recueil de contributions publiées sous le titre certes suggestif: The Manipulation of Literature (1985). Voir aussi J. Lambert dans ce volume. 
d'instrument d'évaluation des théories modernes, ou réduire, au moins, les acquis de celles-ci à des proportions plus modestes que celles qu'elles s'étaient attribuées.

Les jugements de valeur s'avèrent plus intéressants lorsqu'ils sont formulés par les concepteurs et les usagers d'époque, et lorsqu'ils peuvent, le cas échéant, être mis en rapport avec l'information scientifique alors disponible. Il convient de souligner une nouvelle fois l'importance du facteur contextuel, puisqu'en histoire des idées, des jugements de valeur n'ont pas, avec leur objet, un lien détaché de toute influence externe.

L'esquisse suivante veut illustrer comment se constitue l'interrelation entre un jugement de valeur et des facteurs contextuels. Si les grandes synthèses linguistiques de notre siècle ont généralement passé sous silence le phénomène de la traduction, c'est, prétend R. De Beaugrande (1991, pp. 19-27), en partie sous l'influence du comparatisme linguistique qui dominait au siècle précédent: l'usage récurrent du procédé traductif dans la recherche des parentés linguistiques détournait l'attention de la traduction comme objet virtuel d'une étude appropriée. Or, comment une telle attitude eût-elle été possible si, au départ, il n'y avait pas eu partage entre étude linguistique et pratique de la traduction? Pour ce qui est de la France, zone culturelle concernée par cet exemple, ce dispositif avait été légué aux comparatistes sous la forme, portée à son expression radicale par les Idéologues, de l'opposition entre la grammaire en tant que «science» et la traduction en tant que «art». Celui-ci relève donc du domaine des applications, échappe à l'étude scientifique, et subit ainsi, dans l'ordonnance des savoirs, la hiérarchie des valeurs imposée par l'idéologie, discipline-mère sous la plume de Destutt de Tracy, auteur d'Éléments d'idéologie $(1801)^{11}$, lequel exclut délibérément les arts de son propos.

11. «L'homme par sa nature tend toujours au résultat le plus prochain et le plus pressant. Il pense d'abord à ses besoins, ensuite à ses plaisirs. Il s'occupe d'agriculture, de médecine, de guerre, de politique-pratique, puis de poésie et d'arts, avant que de songer à 
Certes, la position subordonnée de la traduction n'est pas une invention du XIX $\mathrm{XIècle}^{\mathrm{e}}$ mais au moins elle se trouve, par le biais du comparatisme, plus solidement cantonnée dans des limites, longtemps incontestées voire transmises à notre siècle, d'une tradition de pensée dominée par la rhétorique. On comprend mieux dès lors le désir latent des contemporains de dérober l'étude de la traduction à ce passé, et de la plier à des critères scientifiques actuels de disciplines dominantes, dans l'espoir d'une revalorisation de la réflexion traductologique. On comprend que celle-ci ait écarté la conception de la traduction comme «art», qu'elle ait fait peu de cas de la riche tradition de l'imagerie traductologique (D'hulst, 1993), laissée pour compte par la linguistique historique, et ensuite par la linguistique structurale ou les études littéraires qui avaient rompu avec une conception stylistique de la rhétorique, la «rhétorique retreinte» aux figures (Genette, 1970).

Ainsi sont cousus, dans les paragraphes qui précèdent - à gros fils, certes - deux dispositifs de valorisation et de dévalorisation d'une étape historique de la réflexion traductologique. Le chercheur les inclura à la liste déjà longue de ses questions ce qui pourrait être désespérant si l'on tient compte du faible nombre d'historiens de la traduction et de la traductologie en particulier conséquence somme toute naturelle de l'amnésie sélective d'une discipline aujourd'hui en pleine expansion. L'histoire et

la philosophie: et lorsqu'il fait un retour sur lui-même et qu'il commence à réfléchir, il prescrit des règles à son jugement, c'est la logique; à ses discours, c'est la grammaire; à ses désirs, c'est ce qu'il appelle morale. Il se croit alors au sommet de la théorie, et n'imagine pas même que l'on puisse aller plus loin. Ce n'est que longtemps après qu'il s'avise de soupçonner que ces trois opérations, juger, parler, et vouloir, ont une source commune; que pour les bien diriger, il ne faut pas s'arrêter à leurs résultats, mais remonter à leur origine; qu'en examinant avec soin cette origine, il y trouvera aussi les principes de l'éducation et de la législation; et que ce centre unique de toutes les vérités est la connaissance de ses facultés intellectuelles.» (Destutt de Tracy, 1970 [1801], pp. xiv-xv) 
l'historiographie méritent un meilleur sort; on doit espérer qu'elles bénéficieront davantage sinon pleinement, cette fois-ci, du retour récemment observé de la perspective historique dans les sciences humaines.

Lieven D'hulst: Institut Supérieur de Traducteurs et Interprètes (RUCA-HIVT), Schildersstraat 41, 2000 Antwerpen.

\section{Références}

BALLARD, Michel (1992). De Cicéron à Benjamin. Traducteurs, traductions, réflexions. Lille, Presses universitaires de Lille.

BERMAN, Antoine (1993). «La traduction et ses discours», in J. Lambert et A. Lefevere, dir. (1993), pp. 31-48.

BLACK, Max (1972). Models and Metaphors. Studies in Language and Philosophy. Ithaca-London, Cornell University Press.

BROECK, Raymond van den (1991). "The Generative Model of the Translation Process"), in K.M. van Leuven-Zwart et T. Naaijkens, dir. (1991), pp. 105-114.

COPELAND, Rita (1991). Rhetoric, Hermeneutics and Translation in the Middle Ages. Academic Traditions and Vernacular Texts. Cambridge, Cambridge University Press.

DE BEAUGRANDE, Robert (1991). "Coincidence in Translation: Glory and Misery Again», Target III(1), pp. 17-53.

DESTUTT DE TRACY, Antoine de (1970 [1801]). Éléments d'idéologie I: Logique. Paris, J. Vrin. 
D'HULST, Lieven (1990). Cent ans de théorie française de la traduction. De Batteux à Littré (1748-1847). Lille, Presses universitaires de Lille.

(1991). «Pourquoi et comment écrire l'histoire des théories de la traduction», Actes du XII congrès mondial de la FIT. Belgrade, Prevodilac, pp. 57-62.

(1993). «Observations sur l'expression figurée en traductologie française (XVIII $-\mathrm{XIX}^{\mathrm{e}}$ siècles)», TTR VI(1), pp. 83111.

(1995). «Enkele stellingen over de historiografie van de vertaalwetenschap», in D. Delabastita et Th. Hermans, dir. Den Haag, Stichting Bibliographia Neerlandica, pp. 7-22.

ELFFERS-VAN KETEL, Els (1991). The Historiography of Grammatical Concepts: 19th- and 20th-century changes in the subject-predicate conception and the problem of their historical reconstruction. Amsterdam-Atlanta GA, Rodopi.

GENETTE, Gérard (1970). «La rhétorique restreinte», Communications 16, pp. 158-171.

HERMANS, Theo, dir. (1985). The Manipulation of Literature. Studies in Literary Translation. London-Sydney, Croom Helm.

HOLMES, James S (1988 [1972]). "The Name and Nature of Translation Studies», in Translated! Papers on Literary Translation and Translation Studies. Amsterdam-Atlanta GA, Rodopi, pp. 76-80.

FOUCAULT, Michel (1966). Les Mots et les choses. Une archéologie des sciences humaines. Paris, Gallimard.

KOERNER, Konrad (1989). Practising Linguistic Historiography. Amsterdam-Philadelphia, John Benjamins. 
KORPEL, Luc (1992). Over het nut en de wijze der vertalingen. Nederlandse vertaalreflectie(1750-1820) in een Westeuropees kader. Amsterdam-Atlanta GA, Rodopi.

KUHN, Thomas (1970 [1962]). The Structure of Scientific Revolutions. Chicago, University of Chicago Press.

LAMBERT, José (1993). «History, Historiography and the Discipline: A Programme» in Y. Gambier et J. Tommola, dir. Translation and Knowledge: SSOTT IV (Scandinavian Symposium on Translation Theory, Turku, 4-6.6.1992). Turku, University of Turku - Centre for Translation and Interpreting, pp. 3-25.

LAMBERT, José et André LEFEVERE, dir. (1993). La Traduction dans le développement des littératures. Bern-Berlin-Frankfurt/MainNew York-Paris-Wien \& Leuven, Peter Lang et Leuven University Press.

LEVÝ, Jiři (1967). "Translation as a Decision Process», in To Honor Roman Jakobson 2. The Hague-Paris, Mouton, pp. 11711182.

LOYSON, Charles (1813). De la manière de traduire les poètes anciens. Paris, Académie impériale ancienne et moderne.

RENER, Frederick M. (1989). Interpretatio. Language and Translation from Cicero to Tytler. Amsterdam-Atlanta GA, Rodopi.

SCHLIEBEN-LANGE, Brigitte (1989). «Überlegungen zur Sprachwissenschaftsgeschichtsschreibung», in Brigitte Schlieben-Lange $e t$ al., dir. Europäische Sprachwissenschaft um 1800. Methodologische und historiographische Beiträge zum Umkreis der «idéologie». Münster, Nodus Publikationen, vol. I, pp. 11-23.

(1991). «Hermeneutik und Serie», in Brigitte Schliebe-Lange et al., dir. Europäische Sprachwissenschaft um 1800. Methodologische und historiographische Beiträge zum Umkreis der «idéologie». Münster, Nodus Publikationen, vol. II, pp. 307-318. 
SECHE, Léon (1905). Lamartine de 1816 à 1830. Elvire et les «Méditations». Paris, Société du Mercure de France.

SNELL-HORNBY, Mary (1988). Translation Studies. An Integrated Approach. Amsterdam-Philadelphia, John Benjamins.

STOLZE, Radegundis (1994). Übersetzungstheorien. Eine Einführung. Tübingen, Gunter Narr Verlag.

SWIGGERS, Pierre (1990). «Reflections on (Models for) Linguistic Historiography», in W. Hüllen, dir. Understanding the Historiography of Linguistics. Problems and Projects. Münster, Nodus Publikationen, pp. 21-34.

VAN LEUVEN-ZWART, Kitty M. et Ton NAAIJKENS, dir. (1991). Translation Studies: the State of the Art. Proceedings of the First James $S$ Holmes Symposium on Translation Studies. Amsterdam-Atlanta GA, Rodopi.

VERMEER, Hans J. (1994). «Translation today: Old and new problems», in Mary Snell-Hornby, Franz Pöchhacker et Klaus Kaindl, dir. Translation Studies: An interdiscipline. AmsterdamPhiladelphia, John Benjamins, pp. 3-16.

RÉSUMÉ: Pour une historiographie des théories de la traduction: questions de méthode - L'historiographie de la traduction et des théories de la traduction est un secteur sousdéveloppé de la traductologie, tant dans la perspective de l'interdisciplinarité que celle-ci réclame et dont on trouve des exemples en particulier dans l'histoire de la discipline que dans l'intérêt grandissant pour une compréhension plus approfondie, en termes historiques, des relations étroites entre les théories modernes spécialement en prenant en compte leurs origines et leur développement. L'article aborde plusieurs problèmes méthodologiques de la recherche historique en théorie de la traduction. En premier lieu, on insiste sur la nécessité de fonder 
l'analyse sur des corpus exhaustifs, ce qui implique de prendre en considération des sources traditionnellement négligées ainsi que les disciplines connexes. En second lieu, on plaide ici en faveur d'une interaction réfléchie entre concepts contemporains et historiques en faisant jouer à ces derniers un rôle complémentaire et non pas de substitut. Les relations entre contenu et contexte peuvent être décrites selon le même principe. En troisième lieu, l'article analyse certains problèmes que posent la nature évolutive des théories et leur constitution en "paradigmes». Enfin, le modèle de progrès prédominant dans les théories contemporaines de la traduction est critiqué et confronté à une perspective sur l'évaluation déterminée par des facteurs historiques proprement dits parmi lesquels le facteur contextuel qui fait ici l'objet d'une analyse particulière.

\section{ABSTRACT: For an Historiography of Translation Theories: Questions of Method - The historiography of translation and of} translation theories is an underdeveloped branch of translation studies, both in view of the latter's claim for interdisciplinarity particularly exemplified by the history of the discipline, and the growing appeal for an in-depth understanding, in historical terms, of the intricate relations between modern theories, esp. taking into account their origins and development. The article deals with several methodological problems of the historical research of translation theories. First, the necessity is stressed to take exhaustive corpora as a basis for the analysis, implying the consideration of traditionally neglected sources, and of correlated disciplines. Secondly, a plea is made for a carefully pondered interaction between contemporary and historical concepts, by understanding the former's role as complementary and not substitutive. The relations between content and context can be described according to the same principle. Thirdly, an analysis is made of some problems involved with the analysis of the evolutionary nature of theories, and their constitution into "paradigms." Finally, the progress-model prevalent in contemporary translation theories is criticized and opposed to a view on evaluation determined by proper historical factors, among which the contextual one receives special emphasis. 